\title{
温度与微结构高度误差对衍射光学元件衍射效率的影响研究
}

\author{
杨亮亮, 赵勇兵, 唐 健, 郭仁甲 \\ （盐城师范学院 物理与电子工程学院, 江苏 盐城 224007)
}

\begin{abstract}
摘要: 基于衍射光学元件的衍射效率与微结构高度误差的关系, 提出了环境温度、微结构高度误差与 衍射效率和带宽积分平均衍射效率的数学分析模型。研究了环境温度变化对带宽积分平均衍射效率的 影响, 分析了工作在一定温度范围内时带宽积分平均衍射效率与相对微结构高度误差的关系。对于工 作在 $8 \sim 12 \mu \mathrm{m}$ 长波红外波段的衍射光学元件, 偏离设计波长越远, 其衍射效率受温度的影响越大。 温度的变化会引起 $100 \%$ 衍射效率对应的峰值相对微结构高度误差发生改变。当衍射光学元件的相对 微结构高度误差在 $\pm 15 \%$ 范围内时, 衍射效率在 $-40^{\circ} \mathrm{C} \sim 80^{\circ} \mathrm{C}$ 的整个温度范围内高于 $91.89 \%$, 带宽积 分平均衍射效率在整个温度范围内高于 $88.58 \%$ 。
\end{abstract}

关键词: 衍射光学; 衍射效率; 微结构高度误差

中图分类号：O436 文献标识码：A 文章编号：1001-8891(2020)05-0213-05

\section{Research on the Influence of Temperature and Microstructure Height Error on Diffraction Efficiency for Diffractive Optical Elements}

\author{
YANG Liangliang, ZHAO Yongbing, TANG Jian, GUO Renjia \\ (School of Physics and Electronics Engineering, Yancheng Teachers University, Yancheng 224007, China)
}

\begin{abstract}
Based on the relationship between diffraction efficiency and microstructure height error for diffractive optical elements (DOEs), mathematical analytical models of environment temperature, microstructure height error, and diffraction efficiency/polychromatic integral diffraction efficiency (PIDE) were put forward. The influence of ambient temperature on the PIDE was researched and the relationship between the PIDE and microstructure height error within a certain temperature range was analyzed. For a DOE working within an 8-12 $\mu \mathrm{m}$ long-waveband infrared range, the influence of temperature on the diffraction efficiency was significant as the wavelength deviated from the designed value. The peak relative microstructure height error corresponding to the $100 \%$ diffraction efficiency changed with the change of temperature. When the relative microstructure height error of the DOE was within $\pm 15 \%$, the diffraction efficiency was higher than $91.89 \%$ in the temperature range from $-40^{\circ} \mathrm{C}$ to $80^{\circ} \mathrm{C}$ and the PIDE was higher than $88.58 \%$ in the entire temperature range.
\end{abstract}

Key words: diffractive optics, diffraction efficiency, microstructure height error

\section{0 引言}

鉴于衍射光学元件具有区别于传统折射透镜的 负色散性质和负热差性质等, 所以, 其广泛应用于各 种成像光学系统中 ${ }^{[1-3]}$ 。衍射光学元件可以直接采用单 点金刚石车削方法进行加工 ${ }^{[4-5]}$ 。，也可以采用光刻技 术或者复制技术 ${ }^{[6-8]}$ 。不管采用哪种加工方法, 都会不 可避免地引入一些加工误差, 而误差的存在会对衍射 光学元件的衍射效率带来直接的影响 ${ }^{[9-12]}$, 进而导致
成像光学系统的像质下降。红外成像光学系统中能够 选择的透镜材料种类有限, 衍射光学元件的出现为红 外系统的设计提供了更多的自由度。致冷型红外系统 要能够在一定的温度范围内实行消热差设计, 所以系 统中采用的衍射光学元件其衍射效率也要进行温度 变化的影响分析。

文献[13]讨论了加工误差对衍射光学元件波前像 差的影响。文献[10]分析了加工误差对带宽积分平均 衍射效率的影响, 指出了微结构高度误差是影响衍射 
效率的重要误差之一。文献[14]给出了环境温度的改 变对谐衍射元件衍射效率的影响。文献[15]给出了考 虑环境温度因素时多层衍射元件衍射效率的优化方 法。本文基于前期工作中给出的微结构高度误差与衍 射效率的数学关系, 建立了衍射效率和温度与相对微 结构高度误差的数学模型。分析了环境温度变化和一 定温度范围内工作时, 微结构高度误差对衍射效率和 带宽积分平均衍射效率的影响。

\section{1 温度与微结构高度误差和衍射效率的理论 关系}

当入射光线从空气介质正入射到单层衍射光学 元件的基底材料上时, 衍射光学元件第 $m$ 衍射级次的 衍射效率为 ${ }^{[11]}$ :

$$
\eta=\sin c^{2}\left\{m-\frac{d_{0}[n(\lambda)-1]}{\lambda}\right\}
$$

式中: $\operatorname{sinc}(x)=\sin (\pi x) / \pi x, m$ 为衍射级次; $d_{0}$ 表示衍 射光学元件的理论微结构高度的大小; $n(\lambda)$ 为元件的 基底材料在波长为 $\lambda$ 时对应的折射率。假设由于加工 引入的微结构高度误差为 $\Delta d$, 那么, 衍射光学元件的 实际微结构高度为:

$$
d=d_{0}+\Delta d=d_{0}(1+\varepsilon)
$$

式中: $\varepsilon=\Delta d / d_{0}$, 表示相对微结构高度误差。考虑微
结构高度误差这一因素后, 式(1)的衍射效率可以表示 为:

$$
\eta_{0}=\sin c^{2}\left\{m-\frac{d_{0}(1+\varepsilon)[n(\lambda)-1]}{\lambda}\right\}
$$

当环境温度变化时, 衍射光学元件的微结构高度 会随之变化。如图 1 所示, 环境温度的改变会引起衍 射光学元件微结构高度大于和小于理论微结构高度。 由温度变化导致微结构高度为 $d_{\mathrm{t}}$, 即:

$$
d_{\mathrm{t}}=d_{0}\left(1+\alpha_{\mathrm{g}} \Delta T\right)
$$

式中: $\alpha_{\mathrm{g}}=\left(1 / d_{0}\right) \mathrm{d} d_{0} / \mathrm{d} T$ 为衍射元件基底材料的热膨胀 系数, 表示温度变化引起的微结构高度的相对改变 量, $\Delta T$ 为环境温度的变化量, 数值上等于环境温度 $T$ 与某一标准温度 (一般为 $20^{\circ} \mathrm{C}$ ) 的差值。

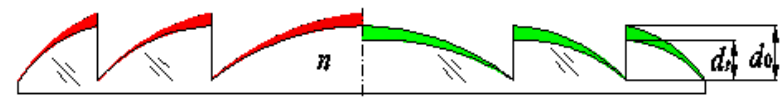

图 1 温度引起微结构高度改变的示意图

Fig.1 Schematic diagram of microstructure height changes caused by temperature

当环境温度变化时, 衍射光学元件的微结构高度 和基底材料的折射率都会发生改变，进一步引起其衍 射效率发生改变。考虑温度这一因素后，衍射光学元 件的衍射效率和温度与相对微结构高度误差的关系 为:

$$
\eta_{m}=\sin \mathrm{c}^{2}\left\{m-\frac{d_{0}\left(1+\alpha_{\mathrm{g}} \Delta T\right)(1+\varepsilon)[n(\lambda)-1]+d_{0}(1+\varepsilon) \frac{\mathrm{d} n}{\mathrm{~d} T} \Delta T}{\lambda}\right\}
$$

当含有衍射光学元件的混合成像光学系统的工作波段范围为 $\lambda_{\text {min }} \sim \lambda_{\text {max }}$ 时, 需要综合考虑衍射光学元件的 带宽积分平均衍射效率, 其与温度和相对微结构高度误差的关系为:

$$
\bar{\eta}_{m}(\lambda)=\frac{1}{\lambda_{\max }-\lambda_{\min }} \int_{\lambda_{\min }}^{\lambda_{\max }} \sin ^{2}\left\{m-\frac{d_{0}\left(1+\alpha_{\mathrm{g}} \Delta T\right)(1+\varepsilon)[n(\lambda)-1]+d_{0}(1+\varepsilon) \frac{\mathrm{d} n}{\mathrm{~d} T} \Delta T}{\lambda}\right\} \mathrm{d} \lambda
$$

\section{2 分析和讨论}

工作在 $8 \sim 12 \mu \mathrm{m}$ 波段的衍射光学元件, 基底材 料采用常用的锗（Ge），衍射级次取 $m=1$, 根据带 宽积分平均衍射效率的最大化计算得到设计波长为 $\lambda_{0}=9.74 \mu \mathrm{m}$, 其微结构高度理论值为 $d_{0}=\lambda_{0} /\left[n\left(\lambda_{0}\right)-\right.$ $1]=3.24 \mu \mathrm{m}$ 。

\section{1 温度变化对衍射效率的影响}

当相对微结构高度误差 $\varepsilon$ 为零时, 根据公式(5), 得到衍射光学元件的衍射效率与波长和温度的关系 如图 2 所示。图 3 给出了在设计波长和边缘波长等几
个不同波长处，衍射效率和温度的变化关系。从图中 可见, 温度从 $-40^{\circ} \mathrm{C}$ 变化到 $80^{\circ} \mathrm{C}$, 在设计波长处, 衍 射效率变化不大; 偏离设计波长越远, 衍射效率受温 度的影响越大。可以看出高于设计波长的几个波长, 如波长为 $12 \mu \mathrm{m}$ 时, 衍射效率随温度的降低而减小, 随温度的升高而增大; 小于设计波长的几个波长, 如 波长为 $8 \mu \mathrm{m}$ 时, 衍射效率随温度的降低而增大, 随 温度的升高而减小。 


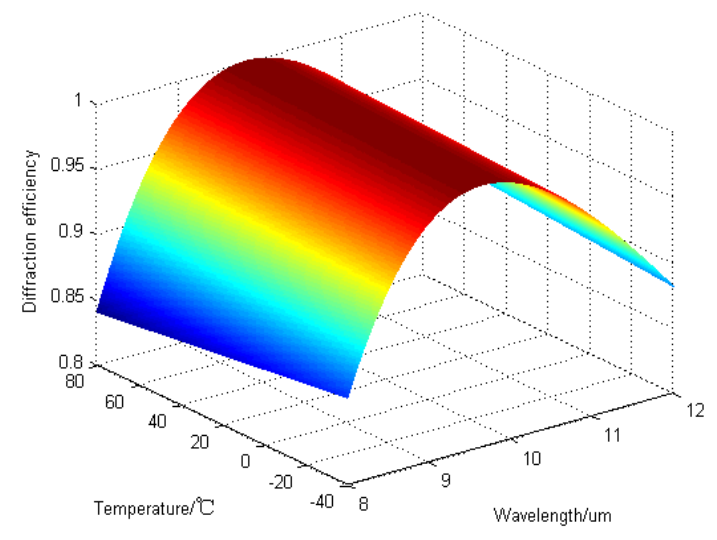

图 2 衍射效率与波长和温度的关系

Fig.2 Relationship of diffraction efficiency, wavelength and temperature

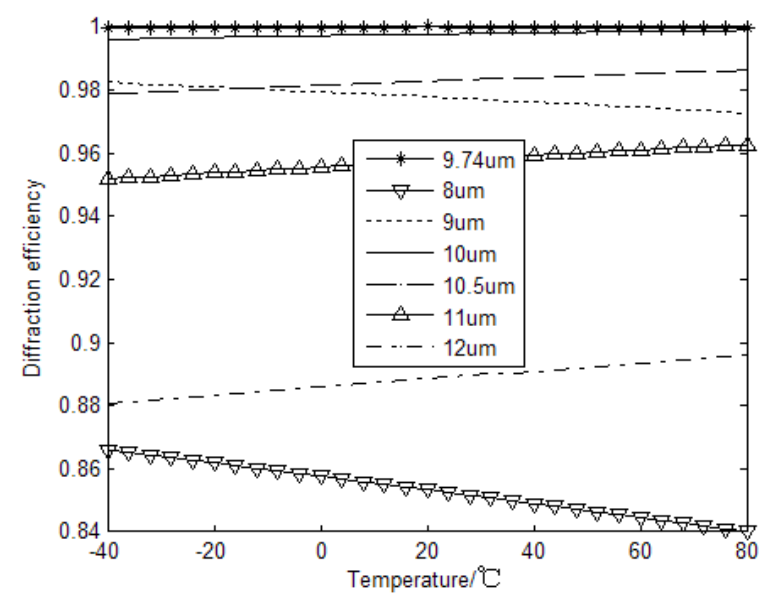

图 3 几个不同波长处的衍射效率与温度的关系

Fig.3 Relationship of diffraction efficiency and temperature at several wavelengths

当环境温度为 $20^{\circ} \mathrm{C} 、-40^{\circ} \mathrm{C}$ 和 $80^{\circ} \mathrm{C}$ 时, 衍射光 学元件的衍射效率随波长的变化曲线关系如图 4 所 示。温度降低时, 实现 100\%衍射效率的峰值波长向 短波移动; 温度升高时, 实现 $100 \%$ 衍射效率的峰值 波长向长波移动。所以, 在一定温度范围内工作的衍 射光学元件, 要注意温度变化时所引起的峰值波长改 变的现象。根据图 3 和图 4 可知在不同温度下对应的 衍射效率, 当环境温度从 $20^{\circ} \mathrm{C}$ 升高到 $80^{\circ} \mathrm{C}$ 时, 整个 波段范围内的最小衍射效率从 $20^{\circ} \mathrm{C}$ 时的 $85.33 \%$ 下降 到为 $84.02 \%$, 下降了 $1.31 \%$; 当环境温度从 $20^{\circ} \mathrm{C}$ 降 低到 $-40^{\circ} \mathrm{C}$ 时, 整个波段范围内的最小衍射效率为 $86.60 \%$, 相比 $20^{\circ} \mathrm{C}$ 时衍射效率, 升高了 $1.27 \%$ 。

\section{2 温度变化对带宽积分平均衍射效率的影响}

当相对微结构高度误差 $\varepsilon$ 为零, 环境温度范围为 $-40^{\circ} \mathrm{C} \sim 80^{\circ} \mathrm{C}$ 时, 根据公式(6)计算得到衍射光学元件 的带宽积分平均衍射效率与温度的关系如图 5 所示。 当环境温度从 $20^{\circ} \mathrm{C}$ 升高到 $80^{\circ} \mathrm{C}$ 时, 或从 $20^{\circ} \mathrm{C}$ 降低到 $-40^{\circ} \mathrm{C}$ 时, 带宽积分平均衍射效率都是仅下降了
$0.02 \%$ 。改变很小。

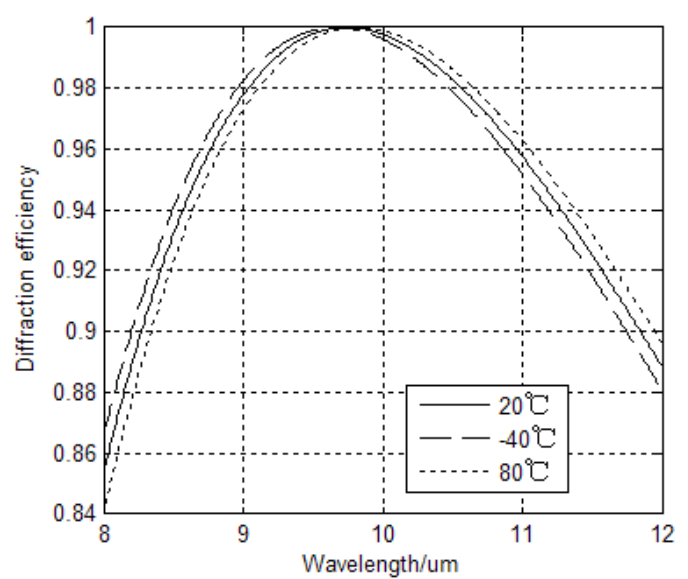

图 4 几个不同温度处的衍射效率与波长的关系

Fig.4 Relationship of diffraction efficiency and wavelength at several temperatures

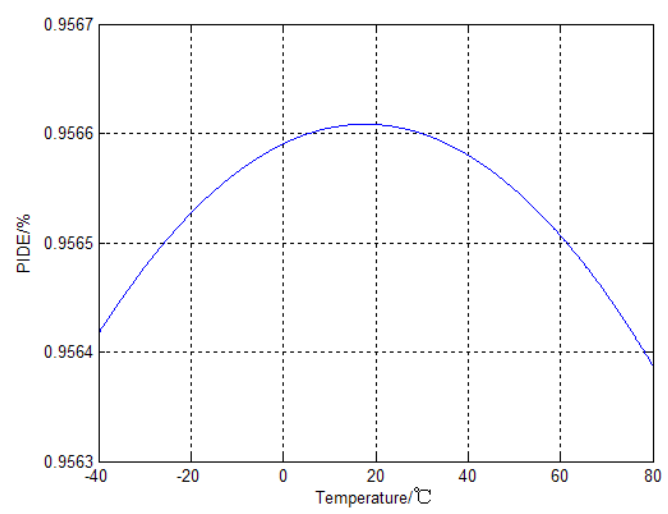

图 5 带宽积分平均衍射效率与温度的关系

Fig.5 Relationship of PIDE and temperatures

\section{3 温度变化和微结构高度误差对衍射效率的影响}

当环境温度为 $20^{\circ} \mathrm{C} 、-40^{\circ} \mathrm{C}$ 和 $80^{\circ} \mathrm{C}$ 时, 利用公 式(5)得到衍射光学元件的衍射效率与相对微结构高 度误差的关系曲线如图 6 所示。对比图 4, 温度降低 时, 实现 100\%衍射效率的峰值相对微结构高度误差 向正值方向移动; 温度升高时，实现 $100 \%$ 衍射效率 的峰值相对微结构高度误差向负值方向移动。所以, 在一定温度范围内工作的衍射光学元件, 要注意温度 变化时所引起的峰值相对微结构高度误差改变的现 象。

当相对微结构高度误差为 0 、 $\pm 5 \%$ 、 $\pm 10 \%$ 和 $\pm 15 \%$ 时, 衍射光学元件的衍射效率与温度的关系曲 线如图 7 所示。可以看出, 当衍射光学元件的相对微 结构高度误差在 $\pm 5 \%$ 范围内时, 衍射效率在 $-40^{\circ} \mathrm{C}$ 到 $80^{\circ} \mathrm{C}$ 的整个温度范围内高于 $98.87 \%$; 当衍射光学元件 的相对微结构高度误差在 $\pm 10 \%$ 范围内时，衍射效率 在 $-40^{\circ} \mathrm{C} \sim 80^{\circ} \mathrm{C}$ 的整个温度范围内高于 $96.13 \%$; 当衍 射光学元件的相对微结构高度误差在 $\pm 15 \%$ 范围内 
时, 衍射效率在 $-40^{\circ} \mathrm{C} \sim 80^{\circ} \mathrm{C}$ 的整个温度范围内高于 91.89\%。

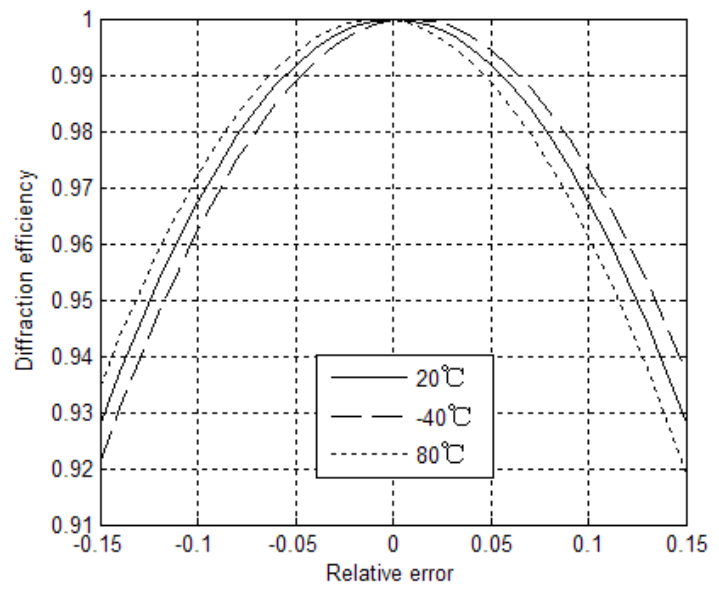

图 6 几个不同温度处的衍射效率与相对微结构高度误差的关 系

Fig.6 Relationship of diffraction efficiency and relative microstructure height error at several temperatures

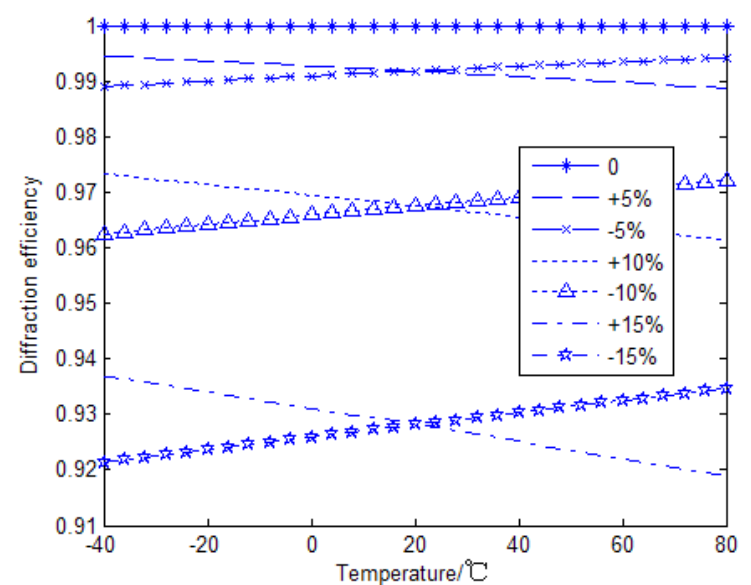

图 7 几个不同相对微结构高度误差处的衍射效率与温度的关 系

Fig.7 Relationship of diffraction efficiency and temperature at several relative microstructure height errors

\section{4 温度变化和微结构高度误差对带宽积分平均衍 射效率的影响}

当环境温度变化范围为 $-40^{\circ} \mathrm{C}$ 到 $80^{\circ} \mathrm{C}$ 时, 利用公 式(6)计算得到衍射光学元件的带宽积分平均衍射效 率与相对微结构高度误差的关系如图 8(a)所示。考虑 到温度的影响, 带宽积分平均衍射效率的最大值为 $95.65 \%$, 此时对应的相对微结构高度误差为 $-0.04 \%$; 当相对微结构高度误差分别为 $-15 \%$ 和 $+15 \%$ 时, 带 宽积分平均衍射效率分别为 $89.19 \%$ 和 $89.41 \%$ 。

当环境温度变化范围相对于 $20^{\circ} \mathrm{C}$ 不对称时, 例如 $-40^{\circ} \mathrm{C}$ 到 $40^{\circ} \mathrm{C}$ 时, 同理, 计算得到衍射光学元件的带 宽积分平均衍射效率最大值为 $95.66 \%$, 对应的相对微 结构高度误差为 $0.24 \%$ 。若环境温度变化范围为一 $20^{\circ} \mathrm{C} \sim 100^{\circ} \mathrm{C}$ 时, 带宽积分平均衍射效率最大值为 95.65\%, 如图 8(b)对应的相对微结构高度误差为一 $0.32 \%$ 。可见当环境温度不对称时, 最大带宽积分平 均衍射效率所对应的相对微结构高度误差会有所区 别。

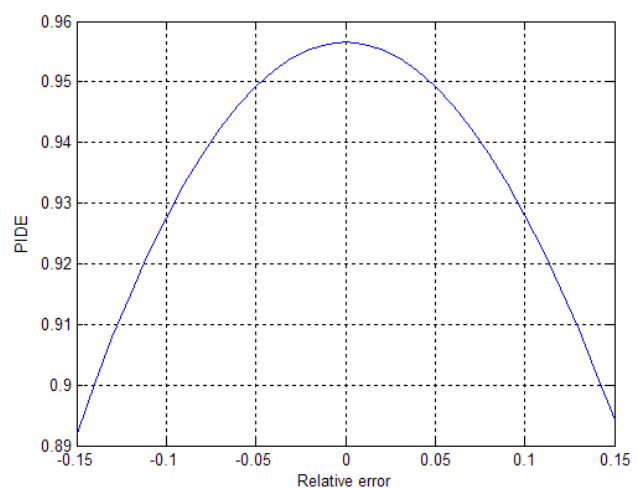

(a) $-40^{\circ} \mathrm{C} \sim 80^{\circ} \mathrm{C}$

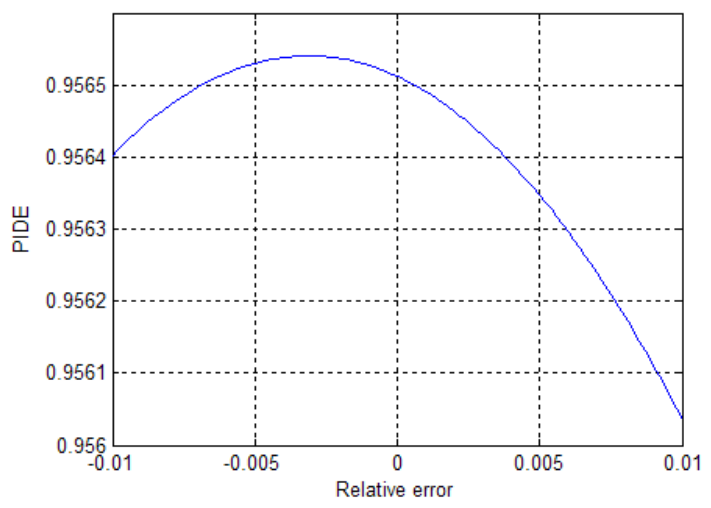

(b) $-20^{\circ} \mathrm{C} \sim 100^{\circ} \mathrm{C}$

图 8 带宽积分平均衍射效率和相对微结构高度误差的关系

Fig.8 PIDE of DOEs versus relative depth-scaling error

当相对微结构高度误差为 $0 、 \pm 5 \%$ 、 $\pm 10 \%$ 和 $\pm 15 \%$ 时, 图 9 给出了衍射光学元件的带宽积分平均 衍射效率与温度的关系。可见, 当衍射光学元件的相 对微结构高度误差在 $\pm 5 \%$ 范围内时, 带宽积分平均衍 射效率在 $-40^{\circ} \mathrm{C}$ 到 $80^{\circ} \mathrm{C}$ 的整个温度范围内高于 94.66\%; 当衍射光学元件的相对微结构高度误差分别 在 $\pm 10 \%$ 和 $\pm 15 \%$ 范围内时，带宽积分平均衍射效率 在整个温度范围内分别高于 $92.28 \%$ 和 $88.58 \%$ 。

\section{3 结论}

基于衍射效率与微结构高度误差的表达式, 建立 了工作在一定环境温度范围内的衍射光学元件的衍 射效率与带宽积分平均衍射效率和温度与相对微结 构高度误差的数学关系表达式。对于工作中 $8 \sim 12 \mu \mathrm{m}$ 长波红外波段的衍射光学元件, 偏离设计波长越远, 其衍射效率受温度的影响越大。温度的变化会引起 $100 \%$ 衍射效率随对应的峰值波长发生改变, 也会引起 
100\%衍射效率所对应的峰值相对微结构高度误差偏 离理想情况。当衍射光学元件的相对微结构高度误差 在 $\pm 15 \%$ 范围内时, 在 $-40^{\circ} \mathrm{C} \sim 80^{\circ} \mathrm{C}$ 的整个温度范围 内衍射效率高于 $91.89 \%$, 带宽积分平均衍射效率高于 88.58\%。该分析方法和结论为工作在一定温度范围内 的衍射光学元件的设计提供了理论基础。

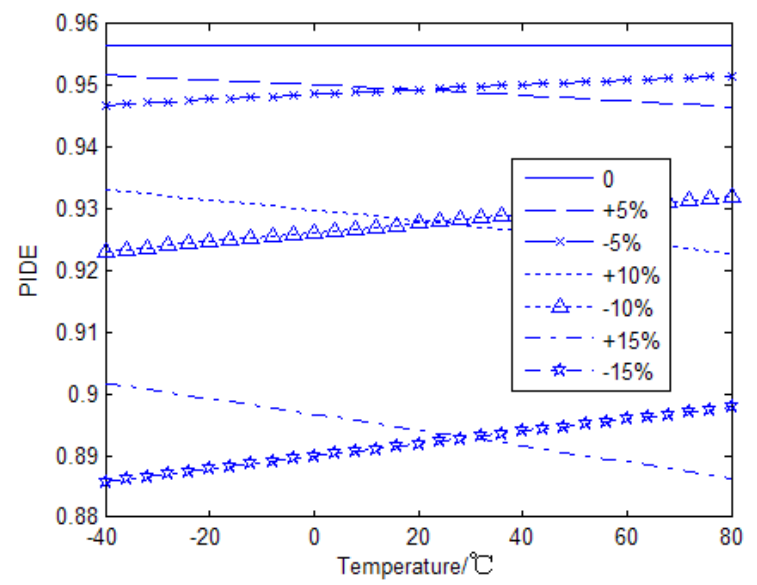

图 9 几个相对微结构高度误差处的带宽积分平均衍射效率与 温度的关系

Fig.9 Relationship of PIDE between temperature at several relative microstructure height errors

\section{参考文献:}

[1] Missig M D, Morris G M. Diffractive optics applied to eyepiece design[J]. Applied Optics, 1995, 34(14): 2452-2461.

[2] 刘秀军, 张金旺, 张华卫, 等. 中波红外制冷型光学系统消热差设计 [J]. 应用光学, 2013, 34(3): 391-396.

LIU Xiujun, ZHANG Jinwang, ZHANG Huawei, et al. Athermal design of cooled MWIR optical system[J]. Journal of Applied Optics, 2013, 34(3): 391-396.

[3] 王吴, 康福增, 赵卫, 等. 一种红外双波段衍射望远镜的光学设计 [J]. 红外与毫米波学报, 2019, 38(1): 39-43.

WANG Hao, KANG FuZeng, ZHAO Wei, et al. An optical design for dual-band infrared diffractive telescope[J]. Journal of Infrared and Millimeter Waves, 2019, 38(1): 39-43.

[4] Wood A P. A hybrid refractive-diffractive lens for manufacture by diamond turning[J]. SPIE, 1991, 1573: 122-128.

[5] C Gary Blough, M Rossi, Stephen K Mack, et al. Single-point diamond turning and replication of visible and near-infrared diffractive optical elements[J]. Applied Optics, 1997, 36(20): 4848-4654.

[6] Kim Younggwang, Rhee Hyuggyo, Ghim Youngsik, et al. Dual-line fabrication method in direct laser lithography to reduce the manufacturing time of diffractive optics elements[J]. Optics Express, 2017, 25(3): 1636-1645.
[7] Kalima V, Pietarinen J, Siitonen S, et al. Transparent thermoplastics: Replication of diffractive optical elements using micro-injection molding[J]. Optical Materials, 2007, 30(2): 285-291.

[8] 程习敏, 白瑜, 谢伟民, 等. 衍射光学元件的加工工艺及其在各种光 谱镜头中的应用 $[J]$. 光电技术应用, 2014, 29(2): 31-38.

CHENG Ximin, BAI Yu, XIE Weimin, et al. Fabrication Technology and Application in Spectral Lens of Diffractive Optical Elements[J]. Electro-Optic Technology Application, 2014, 29(2): 31-38.

[9] 高龙, 薛常喜, 杨红芳, 等. 偏心误差对长波红外波段多层衍射光学 元件衍射效率的影响 [J]. 光学学报, 2015, 35(6): 0623004.

GAO Long, XUE Changxi, YANG Hongfang, et al. Effect of Decenter Errors on Diffraction Efficiency of Multilayer Diffractive Optical Elements in Long Infrared Waveband[J]. Acta Optica Sinica, 2015, 35(6): 0623004.

[10] 毛珊, 崔庆丰. 双层衍射元件加工误差对带宽积分平均衍射效率的 影响[J]. 光学学报, 2016, 36(1): 0105001.

MAO Shan, CUI Qingfeng. Effect on Polychromatic Integral Diffraction Efficiency for Two-Layer Diffractive Optics[J]. Acta Optica Sinica, 2016, 36(1): 0105001.

[11] 杨亮亮, 刘成林, 张志海, 等. 斜入射微结构高度误差的优化设计 [J]. 激光与光电子学进展, 2017, 54(6): 060501.

YANG Liangliang, LIU Chenglin, ZHANG Zhihai, et al. Optimal Design of Depth-Scaling Error with Oblique Incidence[J]. Laser \& Optoelectronics Progress, 2017, 54(6): 060501.

[12] 杨亮亮. 双层衍射光学元件微结构高度的优化设计 [J]. 红外, 2019, 40(1): 11-15.

YANG Liangliang. Optimal Design of the Microstructure Height for Double-layer Diffractive Optical Elements[J]. INFRARED, 2019, 40(1): $11-15$.

[13] Thomas Hessler, Markus Rossi, Rino E Kunz, et al. Analysis and optimization of fabrication of continuous-relief diffractive optical elements[J]. Applied Optics, 1998, 37(19): 4069-4079.

[14] 常笑薇. 环境温度变化对双波段谐衍射光学元件衍射效率的影响 [J]. 北京交通大学学报, 2016, 40(6): 122-126.

CHANG Xiaowei. Relationship between diffraction efficiency and environment temperature change for double wavebands for harmonic diffractive optics[J]. Journal of Beijing Jiaotong University, 2016, 40(6): $122-126$.

[15] PIAO Mingxu, CUI Qingfeng, ZHANG Bo, et al. Optimization method of multilayer diffractive optical elements with consideration of ambient temperature[J]. Applied optics, 2018, 57(30): 8861-8869. 